\title{
A New High Strength High Ductile Nodular Iron
}

\author{
Werner Menk \\ Georg Fischer Automotive AG, CH-Schaffhausen \\ werner.menk@georgfischer.com
}

Keywords: Ductile iron, strength, ductility, solid solution strengthened, SiboDur, strike load, brittleness.

\begin{abstract}
The new solution strengthened ferritic ductile iron grades are well established meanwhile, especially the grade EN-GJS-500-14 is of interest: Compared to the conventional EN500-7 with same tensile strength, from $320 \mathrm{MPA}$ to $400 \mathrm{MPa}$ raised $0.2 \%$ - proof strength and from $7 \%$ to $14 \%$ doubled elongation after fracture are very interesting properties of course and the pure ferritic structure promises a better machinability furthermore. Therefore, a customer of us also substituted a part from steel to EN-GJS-500-14. All calculations showed a very good performance of the parts, but as soon as in serial production field damages occurred in a manner that the parts completely broke without advance warning. The investigation of the damages showed, that the parts are not only statically and cyclically loaded, but also by strikes. Deeper investigations about strike loads confirmed, that EN-GJS-500-14 is not really ductile under these conditions. The reflection to the philosophy we had with our SiboDur-700 concept, namely to combine a moderate solid solution strengthening with a moderate strengthening by pearlite with Copper, brought us to a new high strength high ductile material we call SiboDur-500. Same strength as EN-GJS-500-14, 10 \% lower in $0.2 \%$ - proof stress, slightly lower in elongation after fracture, but double to more than four times energy consumption at strike loads depending on temperature!
\end{abstract}

\section{Introduction}

Years ago, especially in Sweden, a new family of ductile iron materials was propagated: Solid solution strengthened ferritic ductile iron. Since the 2011 edition, three grades are integrated into the European Standard EN 1563: EN-GJS-450-18, EN-GJS-500-14 and EN-GJS-600-10. The introduction of these materials into the European standard generated a large interest of many engineers, which came to a real hype meanwhile. The reason is clear: While GJS-450-18 is not very different from the standard GJS-400-15, especially the grade EN-GJS-500-14 promises to have big advantages compared to the standard grade EN-GJS-500-7. Same tensile strength, from 320 MPA to $400 \mathrm{MPa}$ raised $0.2 \%$ - proof strength and from $7 \%$ to $14 \%$ doubled elongation after fracture are very interesting properties of course and the pure ferritic structure promises a better machinability furthermore. With the higher strength grade EN-GJS-600-10 very early sceptic comments raised, because the Silicon content to reach the required strength is such high, that the risk of an embrittlement of the ferrite even at room temperature and on a tensile test bar is high. But up to now, the grade EN-GJS-500-14 has the reputation to be a high strength and very high ductile material.

Last year, we were very happy therefore that an important customer of us decided to substitute a steel part for large off-road commercial vehicles to EN-GJS-500-14 and we were designed to deliver these parts. All calculations showed a very good performance of the parts, but as soon as in serial production field damages occurred in a manner that the parts completely broke without advance warning.

\section{First Investigations}

The investigation of the damages showed, that the parts are not only statically and cyclically loaded, but also by strikes. Deeper investigations about strike loads confirmed, that EN-GJS-500-14 is not really ductile under these conditions: 

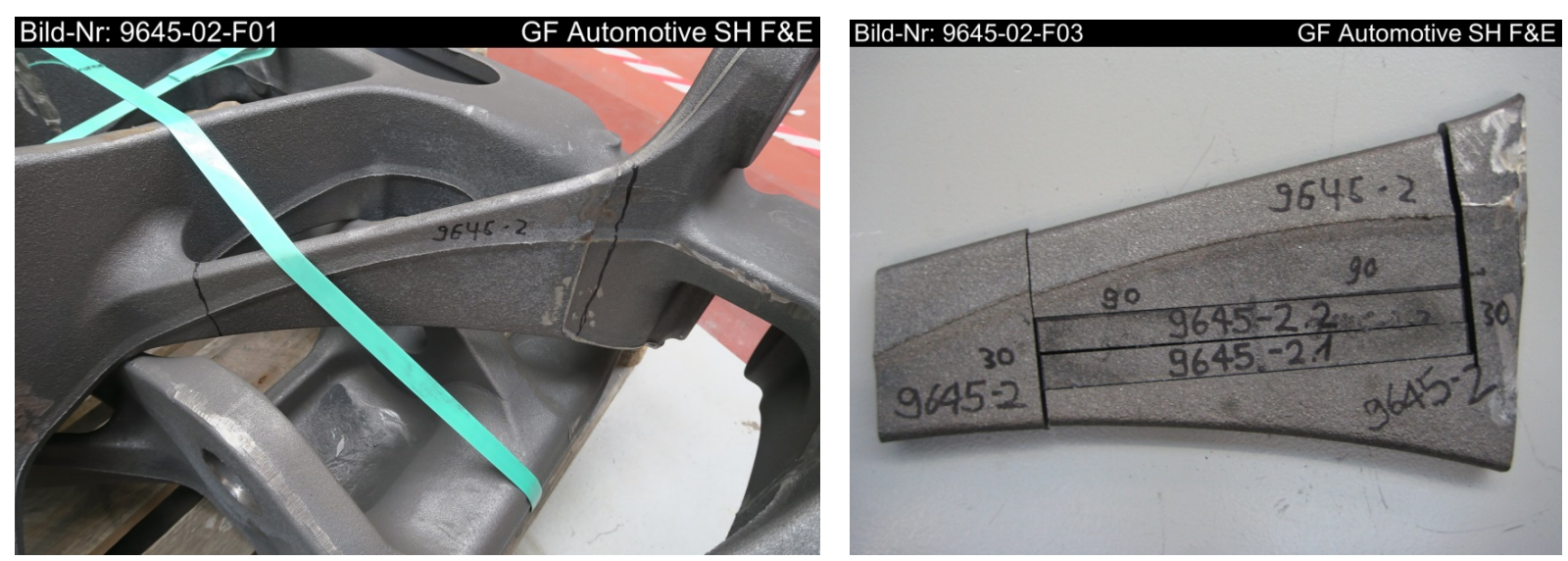

Fig. 1a, 1b: Samples taken out of the castings to be tested for behaviour at consistant strain bending and strikewise bending. Dimensions of samples: Wall thickness 14.8 to $17.5 \mathrm{~mm}$, width grinded to 12. $1 \mathrm{~mm}$, length $180 \mathrm{~mm}$. Important: the crack initiation side of the samples is not machined.

The samples were tested on a hydraulic testing machine for consistant strain with a deformation speed of $5 \mathrm{~mm} / \mathrm{min}$. and with a ,Rosand' testing equipment for instrumented strike load with a deformation speed of $3.6 \mathrm{~m} / \mathrm{sec}$ which equals $216^{\prime} 000 \mathrm{~mm} / \mathrm{min}$.

First tests were done under comparison with the GF material 'SiboDur 700-10' with $700 \mathrm{MPa}$ tensile strength and $10 \%$ elongation after fracture. (SiboDur 700-10 is a GF trademark with a patented composition. This material is not comparable with a ductile iron grade according to EN1563.)

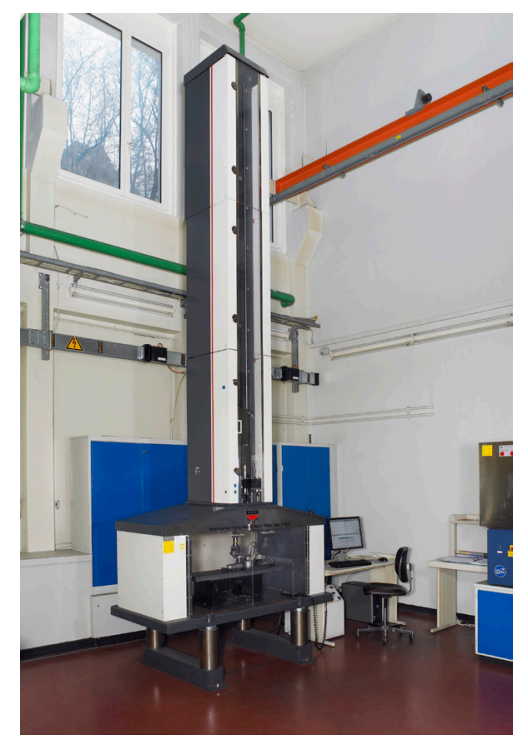

Fig. 2: 'Rosand' testing machine for instrumented testing of strike load conditions. The ,Rosand' testing machine consists of a tower in which a weight of up to $103 \mathrm{~kg}$ mass can be brought into heights of up to $2500 \mathrm{~mm}$. The max. energy available for the strike is 2'700 J therefore. Falling down to the sample, the mass breaks the sample and load and deflection up to breakage can be measured, therefore the energy necessary to break the sample.

The result of these first tests can be seen in Fig. 3. While GJS-500-14 behaves very ductile in the static test, the dynamic test reveals a brittle behavior with very few plastic deformation and much lower energy consumption. The fracture surface also shows a brittle inter- and transcristalline fracture - Fig. 4. The behavior in the dynamic test is very similar to samples of SiboDur-700-10, which is much higher in tensile strength. 


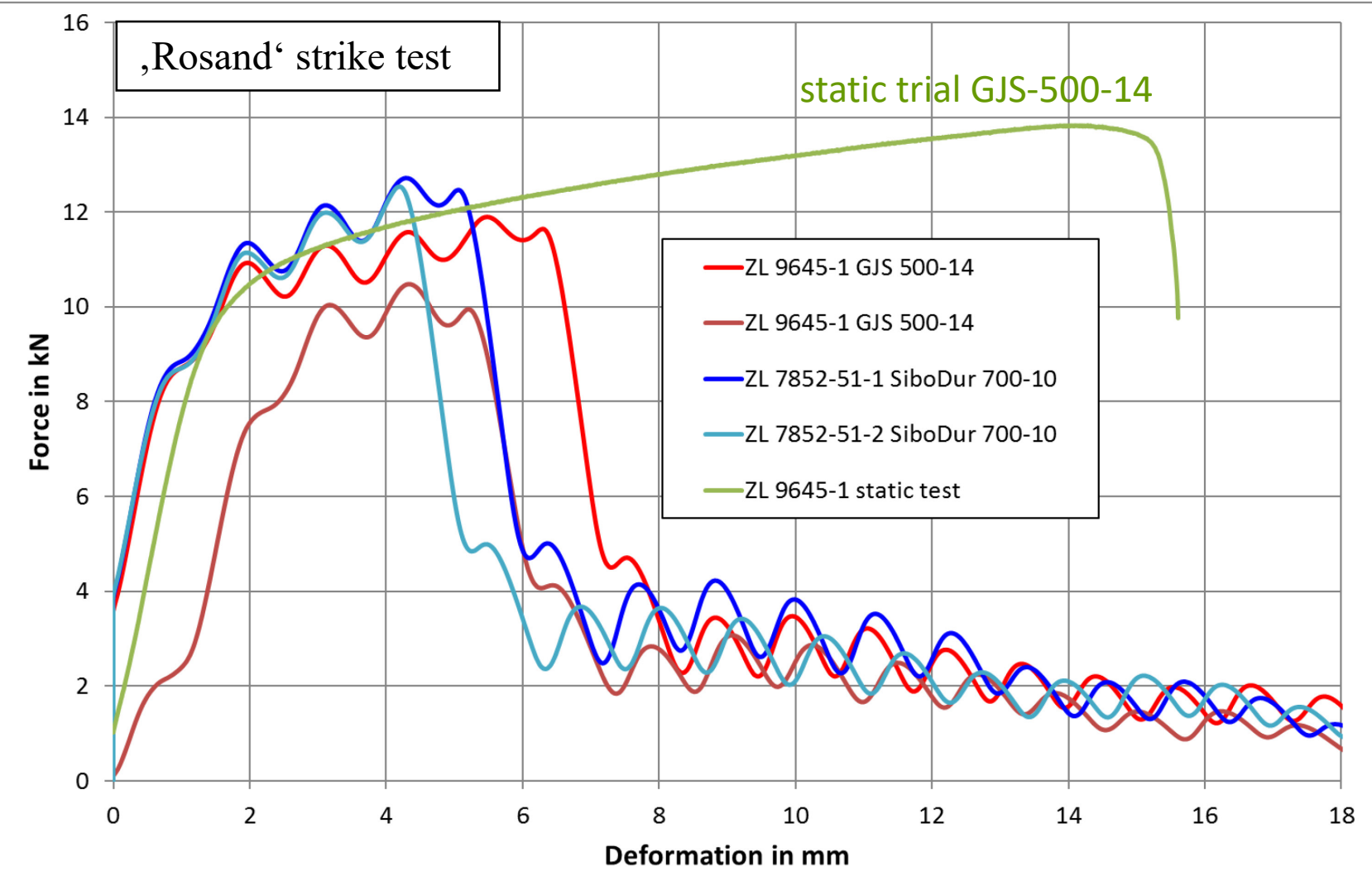

Fig. 3: Result of static and dynamic test of samples GJS-500-14 and SiboDur 700-10: Graph force vs. deformation at room temperature.

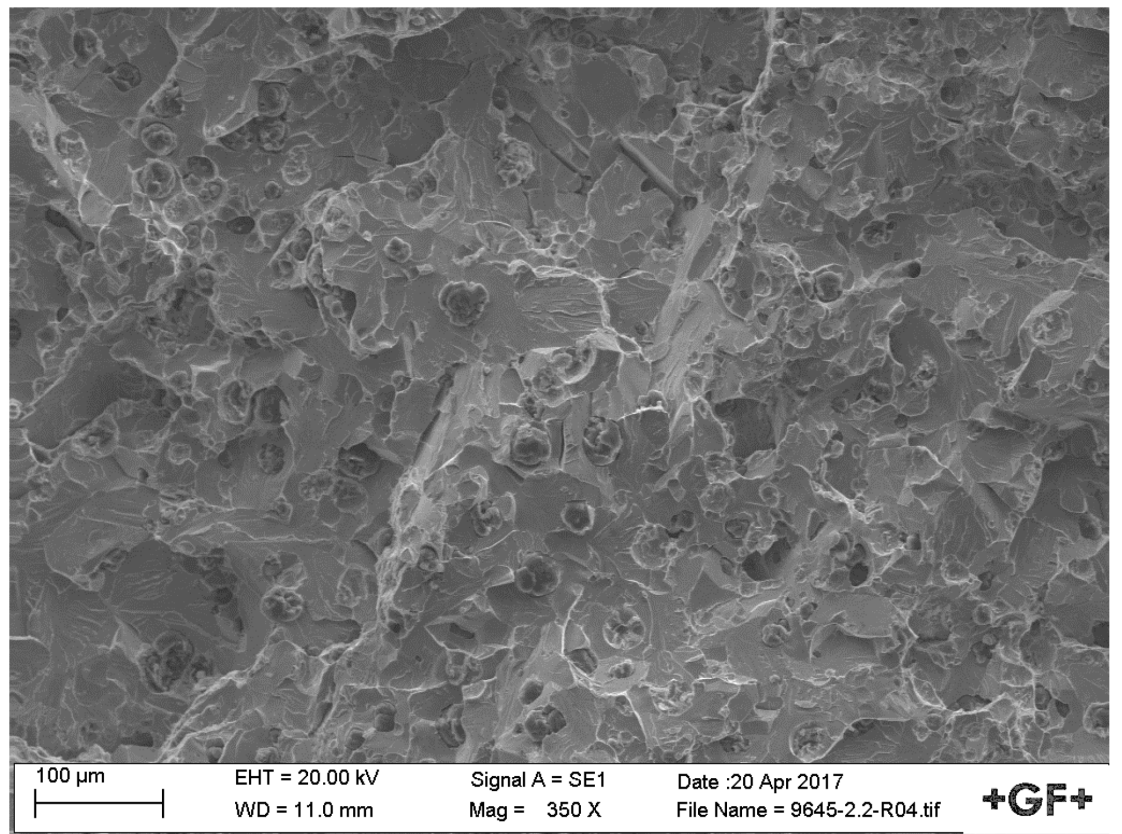

Fig. 4: Brittle fracture of GJS-500-14 after strike test on 'Rosand'- equipment at room temperature (SEM-picture).

\section{A new high strength high ductile nodular iron}

The reflection to the philosophy we had with our SiboDur-700 concept, namely to combine a moderate solid solution strengthening with a moderate strengthening by pearlite with Copper, brought us to a new high strength high ductile material we call SiboDur-500. For the part in question, we chose the following chemical composition: 
$\mathrm{C}=3.5 \%, \mathrm{Si}=2.8 \%, \mathrm{Mn}=0.2 \%, \mathrm{Cu}=0.28 \%, \mathrm{~S}, \mathrm{P}, \mathrm{Mg}$ standard composition

With this composition, the mechanical properties of samples taken out of the part at different positions were:

$\mathrm{Rp} 0.2=330$ to $360 \mathrm{MPa}($ mean $345 \mathrm{MPa})$

$\mathrm{Rm}=500$ to $560 \mathrm{MPa}($ mean $533 \mathrm{MPa})$

$\mathrm{A}=11$ to $15 \%$ (mean $12.8 \%$ )

$\mathrm{HB}=170$ to 200 (mean 186)

Depending on the position, the pearlite/ferrite ratio was 50 to $70 \%$ of ferrite and 30 to $50 \%$ pearlite respectively.

Using the same samples as in the previous trials with the two materials SiboDur 450 and the new SiboDur 500, strike tests were done again. SiboDur 450 is similar to EN-GJS-450-18, but a GF trademark. Fig. 5 shows the result of these tests. They reveal clearly, that the SiboDur 450 material is more ductile than SiboDur 500, but at lower tensile strength of course.

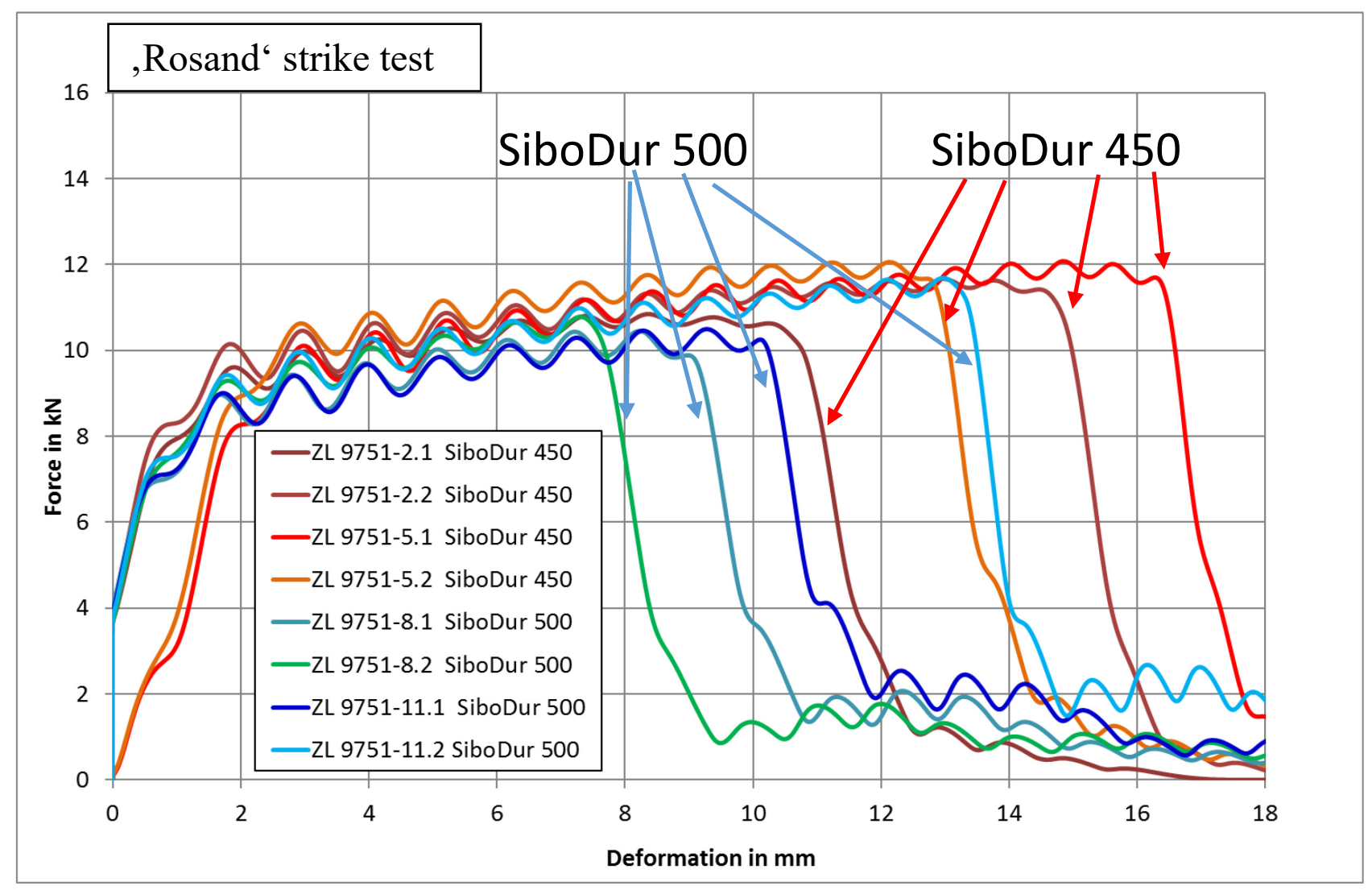

Fig. 5: Result of strike test on 'Rosand' with Sibodur 450 and SiboDur 500 at RT.

For construction parts exposed to impact- or strikewise loads force and plastic deformation are very important. The combination of both values is the energy which is necessary to brake the part. This energy is the integral of the force-deformation curve and can easily be calculated. Fig. 6 shows the summary of all materials tested at room temperature (mean values of force and energy of all samples shown in fig. 3 and 5): 


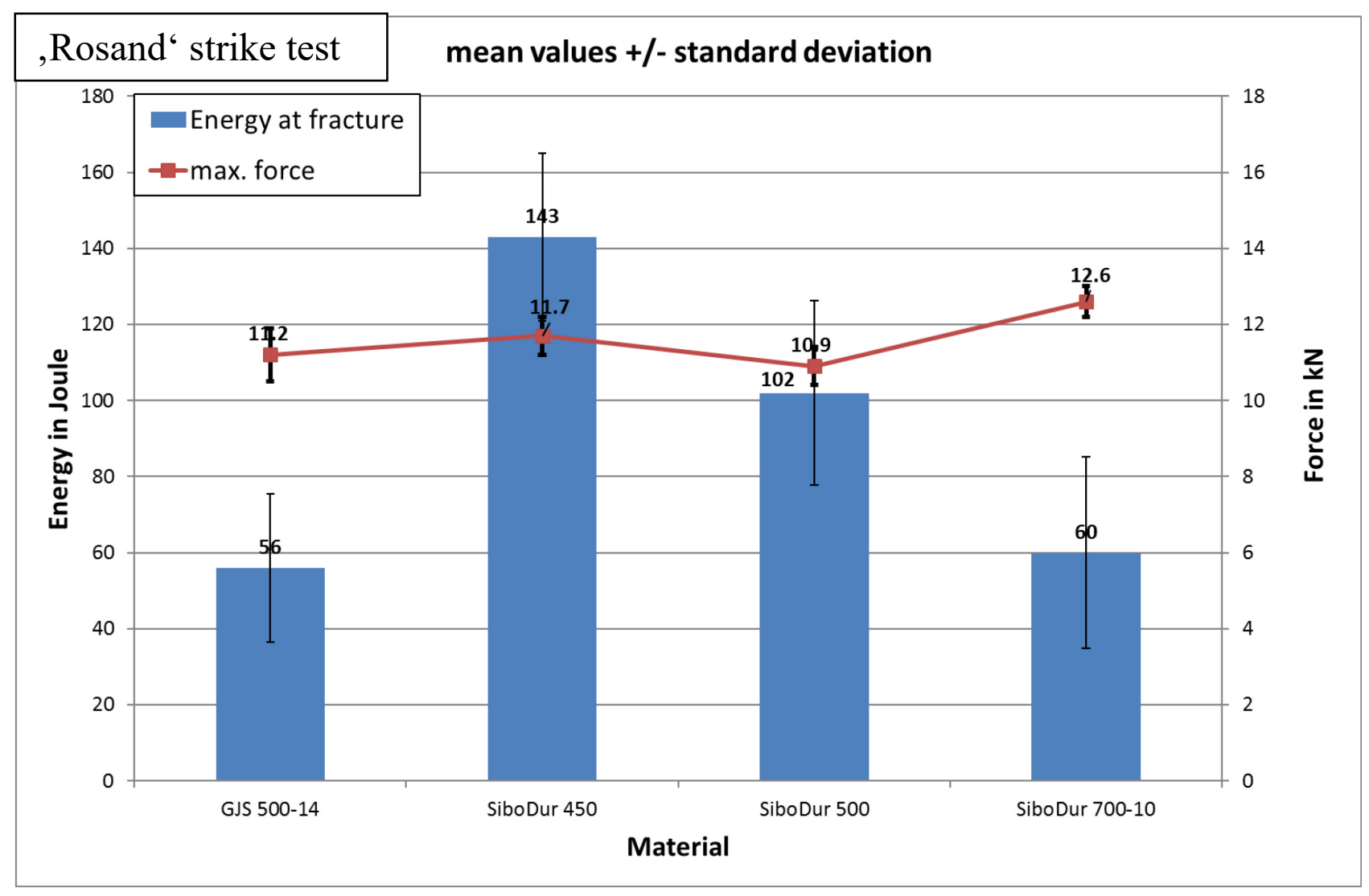

Fig. 6: Summary of tests with 'Rosand' impact tester max. force and total energy.

While SiboDur 700 shows clearly the highest max. force - due to highest strength of this material of course - SiboDur 450 possesses the highest energy consumption until fracture. Despite a lower yield strength compared to GJS-500-10 and SiboDur 500, SiboDur 450 also is higher in max. force due to a strengthening during deformation, see Fig. 5.

\section{Behaviour at lower temperatures}

To check, if the behavior of the different materials is similar at lower temperatures, same strike tests were carried out using samples of GJS-500-14, SiboDur 450 and SiboDur 500 at a temperature of $-40{ }^{\circ} \mathrm{C}$. For these tests the samples were provided with thermocouples each, cooled down to $-78^{\circ} \mathrm{C}$ and placed in the 'Rosand' impact tester. When the temperature reached $-40{ }^{\circ} \mathrm{C}$, the strike was activated. The results of the comparison between GJS-500-14, SiboDur 450 and SiboDur 500 is shown in fig. 7. 


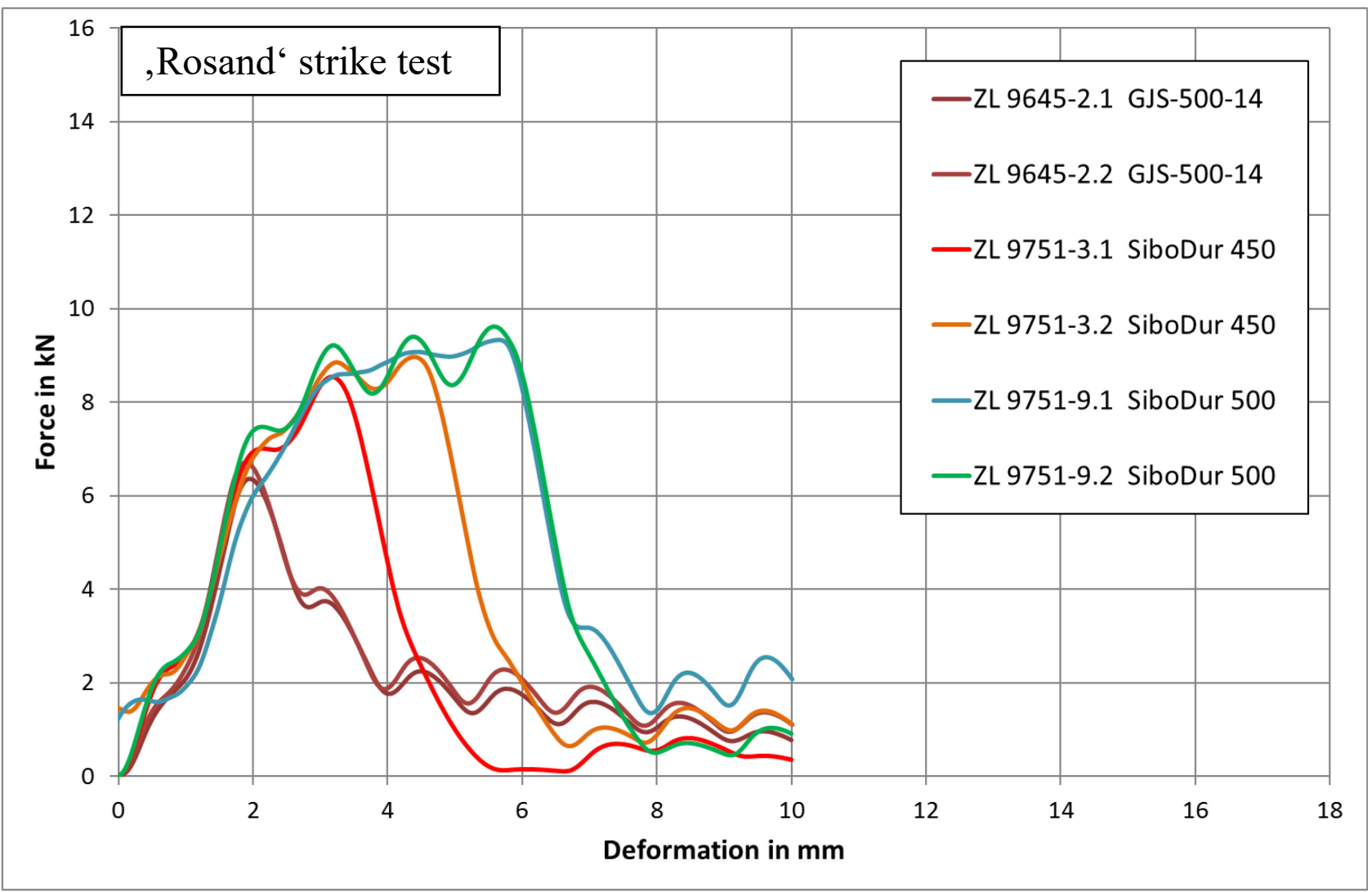

Fig. 7: Result of strike test on 'Rosand' with GJS-500-14, Sibodur 450 and SiboDur 500 at $-40{ }^{\circ} \mathrm{C}$.

Max. force and deformation until fracture of all tested materials are much lower than at room temperature - compare with Fig. 3 and 5 at same scale. As expected from the results at room temperature, the GJS-500-14 material is much weaker as the two SiboDur types. What was not expectable is that the higher strength SiboDur 500 even shows a higher max. force and a higher deformation until fracture than SiboDur 450. This is more clearly shown in Fig. 8, mean values of max. force and energy consumption until fracture.

The explanation for this behavior is not clear, but could be as follows: Silicon content of SiboDur 450 and 500 is about the same (around $2.8 \%$ ). With this Silicon content, there is no embrittlement of both materials in the rim zone at low temperatures as $-40{ }^{\circ} \mathrm{C}$, because both materials are ferritic in the rim zone. But while SiboDur 450 is completely ferritic, SiboDur 500 has a ferritic/pearlitic core zone. This means that in SiboDur 500 the ferritic rim zone is supported by the stronger core zone and can undergo therefore a larger deformation until it starts to crack. 


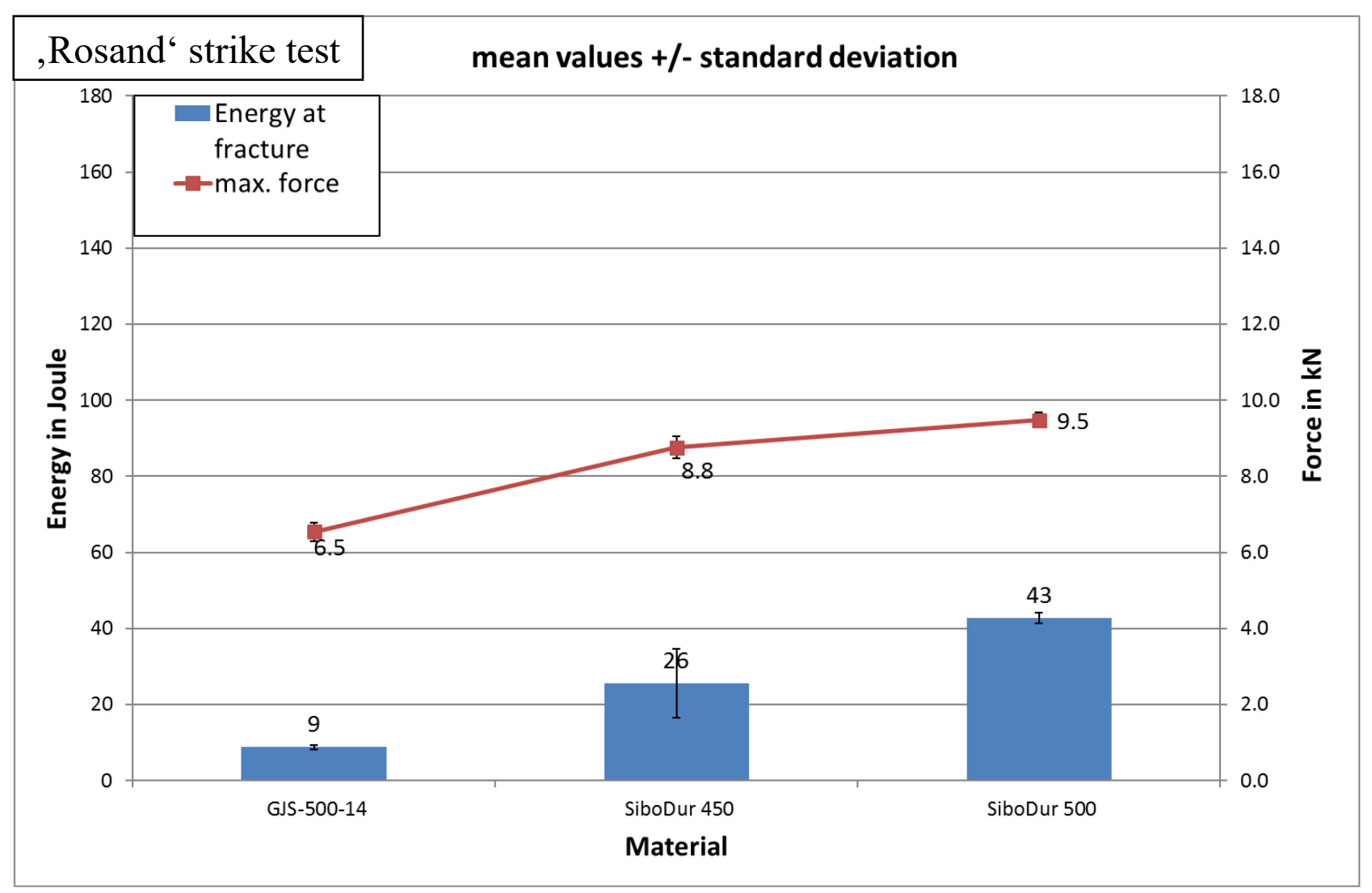

Fig. 8: Summary of tests with 'Rosand' impact tester max. force and total energy at $-40{ }^{\circ} \mathrm{C}$.

\section{Summary}

Silicon alloyed solution strengthened ductile iron as EN-GJS-500-14 is said to be a material with high strength and high ductility at the same time and there is a big demand about this material therefore. This high ductility is given in tensile test bars, tested under standard conditions at slow strain rate. Scared by field failures of castings GJS-500-14, we did tests under impact- or strike conditions on larger samples taken out of real castings and tested with casting skin. The result of these tests showed brittle fractures and low energy consumption until fracture of these samples. The results were comparable to our much higher strength material SiboDur-700-10. The idea was therefore nearby to use the SiboDur-philosophy - slight solution strengthening by Silicon combined with some pearlite by Copper. This led to a material called SiboDur 500 with a much higher ductility at impact- or strike load conditions, but same strength and only slightly lower yield strength and elongation at fracture compared to GJS-500-14.

\section{References}

[1] European patent EP 1270747 B1

[2] European patent EP 1834005 B1 